\section{Heteroauxin Production by Efficient and Inefficient Strains of Rhizobia}

IT has been suggested by Thimann ${ }^{1}$, Link, Wilcox and $\mathrm{Link}^{2}$, and $\mathrm{Link}^{3}$ that $\beta$-indole-acetic acid (heteroauxin) is the agent partially or entirely responsible for the formation of nodules on the roots of leguminous plants. Thimann ${ }^{1}$ advances the hypothesis that the root nodule bacteria convert tryptophane into heteroauxin, $(a)$ by stimulating elongation of a lateral root and $(b)$ by arresting root elongation through continued auxin production, resulting in the formation of a nodule. If this condition exists, there may be differences in the rate of formation and in total amounts of heteroauxin derived from tryptophane, between efficient and inefficient strains of Rhizobia. (Efficient: Rhizobia which infect the host plant and benefit the latter by the symbiosis; inefficient : Rhizobia which infect the host plant but fail to aid in fixing atmospheric nitrogen.) Nodules produced by efficient strains are generally characterized as being large and usually located on the crown of the tap-root, indicating early invasion, while inefficient strains produce very small nodules which are distributed over the secondary root system, indicative of relatively late invasion.

The purpose of these investigations was to determine whether or not early or late invasion could be correlated with rate and amount of production of $\beta$-indole-acetic acid. The substrate upon which the Rhizobia were grown consisted of mannitol 1.0 per cent, $\mathrm{K}_{2} \mathrm{HPO}_{4} 0.05$ per cent, $\mathrm{MgSO}_{4} 0.02$ per cent, NaCl 0.01 per cent, $\mathrm{CaCO}_{3} 0.1$ per cent, $\mathrm{KNO}_{3} 0.05$ per cent and tryptophane ('Pfanstiehl') 0.05 per cent. In contrast to Chen's medium ${ }^{4}$, in which yeast extract was the source of nitrogen, the above medium contained inorganic nitrogen. No $\beta$-indole-acetic acid could be detected in uninoculated controls. Neither could heteroauxin be detected in ether extracts of cultures inoculated in the basal media (same as above with tryptophane omitted), indicating that the bacteria were unable to synthesize the auxin when potassium nitrate was used as a nitrogen source and mannitol as a carbon source.

The basal medium was sterilized at $15 \mathrm{lb}$. pressure for 15 minutes and a solution of tryptophane, sterilized by filtration, added aseptically. Preliminary tests in which 'tryptone' ('Bacto') or peptone ('Bacto') plus 'tryptone' was substituted for tryptophane, yielded data similar to those in the accompanying table, with the exception that the uninoculated controls frequently yielded positive tests for heteroauxin. Therefore, the medium cited above was used exclusively.

FINAI CONCENTRATION OF $\beta$-XNDOLE-ACETIC ACID IN 5 ML. MEDIUM AFTER FOUR WEEKS (AVERAGE OF FOUR EXPERIMENTS).

\begin{tabular}{|c|c|c|c|}
\hline & Efficient & Inefficient & \\
\hline Rh. meliloti & $1: 75,000$ & $1: 10,000$ & \\
\hline Rh. trifolii & $1: 42,000(R)$ & $1: 75,000$ & \\
\hline Rh. leguminosarum & $1: 42,000$ & $1: 45,000(R)$ & \\
\hline $\begin{array}{l}\text { Rh. phaseoli } \\
\text { B. radiobacter }\end{array}$ & $1: 52,000$ & $1: 42,000(R)$ & $1: 10,000(24 \mathrm{hr})$ \\
\hline Controls (uninocula & ted) & 0 & 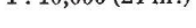 \\
\hline
\end{tabular}

$(R)$ indicates cultures which brought about a rapid conversion.

Cultures were incubated at room temperature for four or five weeks. Presence of heteroauxin was first detected by the Avena technique, but later a colorimetric method ${ }^{5,6}$ was employed to test for indoleacetic acid. Cultures were tested three times weekly, during the course of each experiment, by withdrawing aliquots asceptically. Subcultures into litmus milk at periodic intervals failed to reveal any contamination. The strains studied were $R h$. meliloti cultures
107 (efficient) and 120 (inefficient), Rh. trifolii 202 (inefficient) and 209 (efficient), Rh. leguminosarum 317 (efficient) and 333 (inefficient) and $R h$. phaseoli 403 (inefficient) and 414 (efficient)*.

$B$. radiobacter, commonly found in the root nodules of the Leguminosæ, transformed tryptophane into $\beta$-indole-acetic acid at a more rapid rate than the Rhizobia, yet this organism does not produce nodules on the roots of legumes.

From the data cited, there is no apparent correlation between the rate and amount of indole-acetic acid derived from tryptophane and the ability to fix atmospheric nitrogen by the strains of Rhizobia studied. In fact, there is a tendency for the inefficient to produce auxin more rapidly than the efficient strains. Chen ${ }^{4}$ reports that ineffective strains in most of his experiments produced less growth substance than effective ones. He used $R h$. trifolii only, and our data substantiate his in this respect but not when Rhizobia of other cross inoculation groups were studied. The fact that $B$. radiobacter likewise produces heteroauxin further complicates any interpretation of the data. What significance, if any, can be attached to the production of $\beta$-indole-acetic acid by Rhizobia in the light of the behaviour of $B$. radiobacter?

Dept. of Bacteriology,

Carl E. Georgi.

University of Nebraska.

Albert E. Beguin. Nov. 18.

* Culture numbers refer to the University of Wisconsin strains obtained originally through the courtesy of Messrs. Sarles and Baldwin. 1 Thimann, K. V., Proc. Nat. Acad. Sci., 22, 511-514 (1936).

Link, G. K. K., Wilcox, H. W., and Link, A. DeS., Bot. Gaz., 98, Link, G. K. K , NA .

NATURE, 140, $507(1937)$

${ }^{4}$ Chen, H. K., NATURE, 142, 753 (1938).

${ }^{5}$ Abderhaiden, E., Biochem. Handlexikon, 4, 914 (1911).

Kögl, F., and Kostermans, D. G. F. R., Hoppe-Seyler Z. phys. Chem., 228, 113-121 (1934).

\section{Fossil Man-Apes of South Africa}

By invitation from Dr. Robert Broom, of the Transvaal Museum, Pretoria, and from Prof. Raymond A. Dart, of the Medical School, University of the Witwatersrand, Johannesburg, we visited South Africa in July and August last, and were given every facility for the study of the original fossils described as Australopithecus africanus Dart, 19251, Australopithecus (later Plesianthropus) transvaalensis Broom, $1936^{2}$, and Paranthropus robustus Broom, $1938^{3}$, all probably of Pleistocene age.

Our report on the dentition and dental arches of the South African fossil man-apes has recently been completed and sent to the Pretoria Museum, but the importance of these 'near-men' in future discussions of the origin of man seems to warrant the present brief statement of our principal results.

The dentition of the adult forms described by Broom presents many transitional or annectant conditions between the pithecoid and human stages. The upper canine tooth in a supposed female specimen referred to Plesianthropus transvaalensis has a smaller transverse diameter than the first upper premolar, and is altogether more human than ape-like. Although the upper canine crowns are broken off in the type specimen, the roots prove that these teeth were much smaller than those of apes. A wellpreserved lower canine found in the same cave (at Sterkfontein) is remarkably small in comparison with those of typical modern apes; its crown has a 